\title{
Magnetic properties of as-cast and nanocrystallized $\mathrm{Fe}_{73,5} \mathrm{Cu}_{1} \mathrm{Nb}_{3} \mathrm{Si}_{13,5} \mathrm{~B}_{9}$ ribbons
}

\author{
M.-L. Soltani ${ }^{1^{*}}$, A. Mansri ${ }^{1}$, and M. Lahoubi ${ }^{1}$ \\ ${ }^{1}$ Laboratoire de Physique du Solide, Département de Physique, Université d'Annaba-Annaba 23000
}

\begin{abstract}
This work concerns the study of soft iron-based magnetic materials. More specifically, concerning based iron amorphous materials, it will be done by combining structure and magnetic properties while acting on various parameters, by annealing to characterize the nanocrytallization. An experimental approach of magnetic properties is applied to the amorphous or nanocrystalline ultra soft materials evaluation of power losses, permeability as properties of the cycle of hysteresis. According to the scale of constraints and the nature of heat treatment (without magnetic field or magnetic field), it is induced an anisotropy which changes the magnetization properties. Thus, for ferromagnetic amorphous alloys, the anisotropy induced constraints is eliminated giving rise to a uniaxial anisotropy. FeBSi amorphous type materials can therefore, after introduction of copper (multiplication of germs) and niobium (growth inhibition), get nanocrystallized compositions of $\mathrm{Fe}_{73,5} \mathrm{Cu}_{1} \mathrm{Nb}_{3} \mathrm{Si}_{13,5} \mathrm{~B}_{9}$ after annealing around of $500-600{ }^{\circ} \mathrm{C}$.
\end{abstract}

\section{Introduction}

Polycrystalline solids with grain size less than $100 \mathrm{~nm}$ are called nanocrystalline materials and can be produced using various methods and different starting phase: vapor (inert gas condensation, sputtering, plasma processing, vapor deposition), liquid (electrodeposition, rapid solidification) or solid (mechanical alloying, severe plastic deformation, spark erosion) [1-3], directly in one process, or indirectly through an amorphous precursor. Nanocrystallization of metallic glasses is an example of the second procedure. Two steps are necessary to produce the nanocrystalline material: (1) formation of amorphous state, by quenching of liquid alloy, and (2) partial or complete crystallization of the amorphous alloy by annealing. Among the type of materials, we can distinguish the magnetically soft Fe-based alloys ribbon containing Niobium and cupper results in good soft magnetic properties of 1.2 to 1.4 Tesla for saturation induction $\mathrm{B}_{\mathrm{s}}$ and $10 \times 10^{4}$ for effective permeability at $f=$ $1 \mathrm{kHz}[4-9]$. The properties of magnetically soft Fe-based alloys are sensitive to material structure. So, the diameter of crystallite D should be smaller than the magnetic exchange length in the crystalline phase to reduce the contribution of magnetocrystalline anisotropy of this phase to the total magnetic anisotropy of a material [10].

We consider the study of structure of rapidly solidified magnetic ribbons presenting generally a very high susceptibility and reduced magnetic losses. To achieve this goal, this work relates to layers of soft magnetic materials (coercivity lower than $10 \mathrm{~A} / \mathrm{m}$ ).
Particularly, the $\mathrm{Fe}_{73,5} \mathrm{Cu}_{1} \mathrm{Nb}_{3} \mathrm{Si}_{13,5} \mathrm{~B}_{9}$ ferromagnetic alloy composition so-called Finemet, discovered in 1988 is studied for these outstanding soft magnetic properties [4].These properties are linked to nanocrystalline state, consisting of magnetic nanograins $\alpha-\mathrm{Fe}(\mathrm{Si})$ dispersed in a magnetic amorphous matrix. To obtain these nanostructures, we used, for the first time as-quenched $\mathrm{Fe}-(\mathrm{Cu})-\mathrm{Si}-\mathrm{B}-(\mathrm{Nb})$ alloys. This study aimed at developing enhanced magnetic properties through annealing. The magnetization, coercive force, magnetostriction and magnetic losses were measured at room temperature in rapidly solidified ribbons (amorphous state) and annealed ribbons (nanocrystalline state in an amorphous matrix). Optimum annealing conditions can be found.

\section{Experimental}

Experiments were made on nanocrystalline ribbons. The material used is $20 \mu \mathrm{m}$ thick in $25 \mu \mathrm{mm}$ wide ribbon and alloys are amorphous as received. These ribbons come in toroidal shape facilitating magnetic measurements. Ribbons were produced by rapid quenching using a single roller technique in air. It has connected a magnetic circuit as a transformer, taking $\mathrm{B}(\mathrm{t})$ of the side secondary and $\mathrm{H}$ $(\mathrm{t})$ of the side primary values simultaneously. These two quantities will be our main parameters. Hysteresis loop surface is proportional to the energy dissipated by currents induced in a cycle. The behavior of magnetic material under dynamic regime up to $100 \mathrm{kHz}$ frequency was studied. Hysteresis cycles were plotted for different values of maximum magnetic field and frequency by

\footnotetext{
${ }^{*}$ Corresponding author: ml_soltani@yahoo.com
} 
means of fluxmetric method [11]. Heat treatment conditions start to $375^{\circ} \mathrm{C}$ up to $700^{\circ} \mathrm{C}$ with an increment of $25^{\circ} \mathrm{C}$. The primary interest of material annealing under magnetic field is to modify the shape control of hysteresis loop. This procedure allows you to provide, for purposes of industrial applications with special uses [12]. In the case of nanocrystalline materials, annealing is achieved in nanocrystallisation stage. Annealing of improvement may be carried out otherwise.

\section{Results and discussion}

Fe-based Finemet ${ }^{(}$are nanocrystallizing metallic glasses and do not require any special heat treatment to be converted into nanocrystalline material. They used to be transformed from amorphous to partially nanocrystalline state during simple isothermal annealing at temperature close to crystallization temperature in a time, of usually, $1 \mathrm{~h}$. This kind of treatment can be called conventional nanocrystallization and is widely by many researchers [13]. Both techniques of conventional nanocrystallization are effective only for those glasses which nanocrystallize more easily. In the case of nanocrystallizing Finemet-type alloys [14, 15]. Both high temperature annealing as well as conventional annealing led to similar nanocrystalline structure. Nevertheless, the mean size of nanocrystallites was slightly smaller after high temperature crystallization. This last technique is most often realized using Joule heating and is called flash or current annealing. High temperature crystallization of metallic glasses using Joule heating was applied by many researchers to modify structure and to improve properties of nanocrystalline materials $[16,17]$.

Particularly, larger permeability (by $\sim 50 \%$ ) was found. The change of composition in primary crystals with annealing time was reported; the decreasing lattice parameter $a$ and the increasing curie temperature $\mathrm{T}_{\mathrm{c}}$ indicate a significant change in the composition with increasing size of primary nanocrystals $[18,19]$. Barquin et al observed that composition stabilization of $\mathrm{Fe}(\mathrm{Si})$ nanocrystals, during crystallization process of finemet glass, was achieved after $1 \mathrm{~h}$ annealing at $773 \mathrm{~K}$. To determine susceptibility to nanocrystallization, especially of new alloys, it is useful to correlate microstructure and physical properties (magnetic or mechanical) with thermal properties of glass [20].

\subsection{Effect of annealing on hysteresis loop (magnetization process)}

Magnetic application products require controlling hysteresis loop shape for magnetic material [21]. Induced magnetic anisotropy change in field annealing temperature and time can be controlled [22]. We are focused on an annealing under longitudinal field sample for a uniaxial anisotropy [23, 24] ensuring the presence of walls $180^{\circ}$ parallel to the axis of the Ribbon. An annealing under longitudinal field (magnetic field parallel to a magnetic path direction, i.e., to a ribbon axis) very strongly promotes the emergence of large walls to 180 degrees. The thus induced uniaxial anisotropy manifests itself with a rectangular B-H hysteresis loop, showing a simple mechanism of movement of walls and giving high remanence ratio. An annealing under transverse field (magnetic field parallel to the ribbon width direction) induces a perpendicular to the applied field anisotropy. The mechanism involved is rotations of moments. The shape of hysteresis loop will be rounded and gives a medium remanence ratio. The moments align gradually along the applied field direction. A no field anneal material will have a somewhat rounded appearance with low remanence ratio. In this case, two processes are present: walls movement and rotation of moments. The figure 1 shows a magnetization process measured for both heat treatment and thermo magnetic treatment. Nanocrystalline material annealed under longitudinal field has a very high permeability $>10^{5}$. Figure 1 shows the quasi static hysteresis loop at magnetization saturation of the sample estimated about $1.3 \mathrm{~T}$.

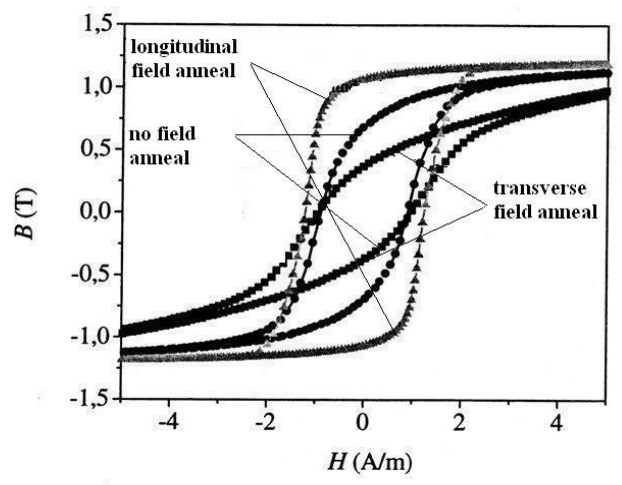

Fig.1 Magnetization loops of the nanocrystalline sample $(\bullet)$ no field anneal, (ם) anneal in a transverse magnetic field, $(\boldsymbol{\Delta})$ anneal in a longitudinal magnetic field

\subsection{Effect of annealing on microstructure}

According to random anisotropy model, the D should not exceed about $15 \mathrm{~nm}$ in the case of $\alpha-\mathrm{Fe}(\mathrm{Si})$ and $\alpha-\mathrm{Fe}$ nanocrystals, present in Finemet [4]. Theses alloys have a primary crystallization and are multicomponent. Silicon and boron are so called glass-forming elements, which facilitate the forming of amorphous state and increase thermal stability of amorphous phase. Iron is a basic constituent of magnetic alloys with magnetic moments. Copper is the responsible for nanocrystalline structure. It was found that formation of initial $\alpha$-FeSi nanocrystals from amorphous material occurs at about $\mathrm{T}=650 \mathrm{~K}$. After conventional annealing for 1 hour, nanocrystalline structure, with average grain diameter $\sim 11 \mathrm{~nm}$, is detected only in the alloys containing pairs of alloying elements (i.e. copper and niobium) [25]. Niobium which is rejected from a crystallization front, to the amorphous matrix, decreases crystal growth because of relatively smaller diffusivity of big atoms of these elements in an amorphous phase [26]. Average diameter of $\alpha-\mathrm{Fe}(\mathrm{Si})$ nanocrystals does not exceed $15 \mathrm{~nm}$ after annealing (for $1 \mathrm{~h}$ ) at temperature up to $900 \mathrm{~K}$ [27].

We can see, in figure 2, the Transmission Electron Microscopy micrographs realized on a classical FINEMET sample annealed at $520^{\circ} \mathrm{C}$, corresponding to a first stage of crystallization $(\sim 800 \mathrm{~K})$ but the use of long time annealing allows obtaining a similar grain size distributions with average grain diameter of about $10 \mathrm{~nm}$. 
We cover two phases: the nanocrystalline phase is composed primarily of iron-silicon $\left(\mathrm{Fe}_{80} \mathrm{Si}_{20}\right)$ and amorphous phase containing the other constituents $\mathrm{FeCuNbB}$. The possibility of controlling the crystalline phase volume fraction can be useful for controlling of magnetostriction in nanorystalline materials $\lceil 20\rceil$.

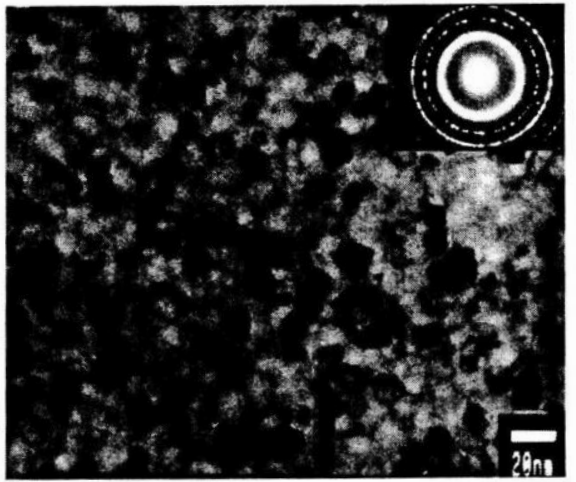

Fig. 2 TEM micrographs and selected-area diffraction patterns of a FINEMET sample annealed at $520^{\circ} \mathrm{C}$

\subsection{Effect of annealing on coercivity}

Performances of these materials are based on their very particular crystalline structure: observation of two phases, one consisting of grains, with nanoscopic size, which realize randomized crystalline orientations. Between grains remains a residue of amorphous phase, which makes the continuity of magnetic exchange between grains. Magnetic properties of the medium result thus from averaging effect on all grains seen by a magnetic wall, phenomenon described qualitatively by random anisotropy model [10]. The practical consequence is the vanishing of the effective magneto-crystalline anisotropy in the walls, and as result of a very low coercivity $\mathrm{H}_{\mathrm{c}}$ and a very high permeability $\mu$. This model gives a good description of the behavior of the coercivity by depending

We can observe, in figure 3 , the influence of annealing temperature on the reducing of coercivity which is the characteristic of soft materials and leads to reducing the core loss. Of course, heat treatment under longitudinal field has designed to make the uniaxial anisotropy and improve hysteresis loop characteristics. Therefore it is expected to an evolution of $\mathrm{B}_{\mathrm{S}}, \mathrm{H}_{\mathrm{C}}$ and $\mu$. Figure 3 enables to observe a step between $450^{\circ} \mathrm{C}$ and $525^{\circ} \mathrm{C}$, beginning of the first stage of nanocrystallization. We can detect a low change of the $\mathrm{H}_{\mathrm{c}}$ coercivity. A second step reveals a reduction of its value near $575^{\circ} \mathrm{C}$. Final step corresponds to a beginning of full nanocrystallization, probably with significant growth of grain size also planned. This allows to estimate the size of grains for each temperature Ta annealing, using the model of Alben [28], Herzer gives a relationship $\mathrm{H}_{\mathrm{C}} \sim \mathrm{D}^{2 / 3}$ using a one dimension random anisotropy model [10]. This gives nanoscopic size 10 to $20 \mathrm{~nm}$ for thermal treatments from 600 to $625^{\circ} \mathrm{C}$ annealing range.

\subsection{Effect of annealing on magnetostriction}

As expected, the influence of annealing on magnetostriction can be given by comparison to coercivity. The longitudinal magnetostriction was measured by a strain gauge attached to the surface ribbon

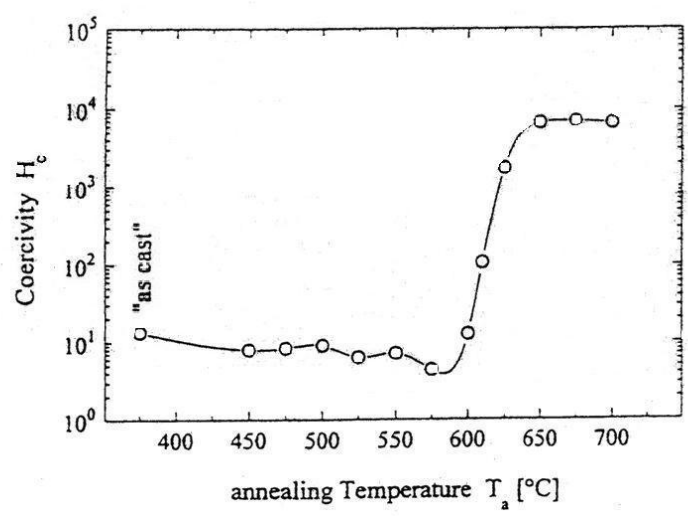

Fig.3 Influence of annealing temerature on coercivity of FINEMET $\mathrm{Fe}_{73.5} \mathrm{Cu}_{1} \mathrm{Nb}_{3} \mathrm{Si}_{13.5} \mathrm{~B}_{9}$

To obtain a constraint relaxation, the reduction is reached with magnetostriction around $\mathrm{T}_{\mathrm{a}} \sim\left[500-600^{\circ} \mathrm{C}\right]$ and saturation magnetostriction $\lambda_{\mathrm{s}}$ can be strongly reduced from $25 \mathrm{ppm}$ to reach about 3 to $1 \mathrm{ppm}$ (see figure 4). The characteristic value is lower than $0.5 \mathrm{ppm}$. This low value of $\lambda_{\mathrm{s}}$ is explained by the fact that amorphous phase and phase crystalline have magnetostriction coefficients with opposed signs, the assembly of two phases gives a material with a low macroscopic magnetostriction.

The total saturation magnetostriction $\lambda_{\mathrm{s}}$ is given by: $\lambda_{s}=V_{F e S i} \cdot \lambda_{s}^{F e S i}+\left(1-V_{F e S i}\right) \cdot \lambda_{\mathrm{s}}^{\mathrm{am}}$, where the $\mathrm{V}_{\mathrm{FeSi}}, \lambda_{\mathrm{s}}^{\mathrm{FeS} i}$ and $\lambda_{\mathrm{s}}^{\mathrm{am}}$ denote the volume fraction of bcc-Fe-Si phase, the saturation magnetostriction of bcc-Fe-Si phase and the saturation magnetostriction of amorphous matrix phase, respectively [29-31]. The $\lambda_{\mathrm{s}}^{\mathrm{FeSi}}$ of FeSi is $-5 \times 10^{-6}$ (Fe-20 atm $\% \mathrm{Si}$ ) and the $\lambda_{\mathrm{s}}^{\mathrm{am}}$ is about $10-25 \times 10^{-6}$ of the positive value. Thus, the increasing $\mathrm{V}_{\mathrm{FeSi}}$ by crystallizing the amorphous alloys leads to decreasing $\lambda_{\mathrm{s}}$. When annealing temperature is higher than crystallization temperature the $\lambda_{\mathrm{s}}$ decreases.

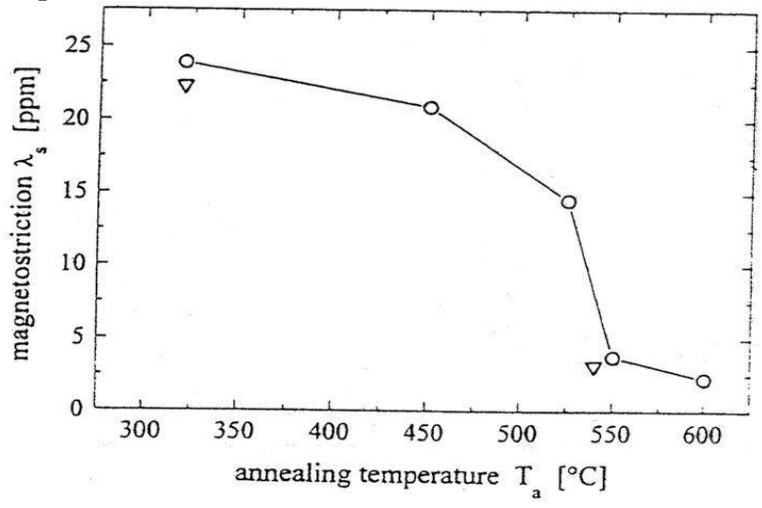

Fig.4 Influence of annealing temperature on magnetostriction of FINEMET $\mathrm{Fe}_{73.5} \mathrm{Cu}_{1} \mathrm{Nb}_{3} \mathrm{Si}_{13.5} \mathrm{~B}_{9}$

The uniaxial anisotropy induced by constraints is in addition to the anisotropy induced by an annealing under longitudinal field. It is distributed on the volume of the sample and is proportional to $\lambda_{\mathrm{s}}$ and $\sigma$ as given by:

$K_{e l}=(3 / 2) x\left|\lambda_{s} . \sigma\right|$ where $\sigma$ is an internal stress. The decrease of magnetostriction by annealing under magnetic field allows obtaining preferentially uniaxial anisotropy. 


\subsection{Analysis of losses}

Evaluation of losses is immediately estimated by hysteresis loop surface and integrated for each value of $\mathrm{B}_{\max }, \mathrm{f}, \mathrm{H}_{\max }$. A calculus program gives loss values in $\mathrm{mW} / \mathrm{kg}$ unit. Losses can be given by $\mathrm{P}(f)$ that is nonlinear in $f$. preferentially we plot $\mathrm{P}(f) / f$ that is a loss by cycle given in $\mathrm{J} / \mathrm{m}^{3}$. In figure 5 , we can see the plots of $\mathrm{P}(f) / f$ versus $\mathrm{B}_{\max }$ in a frequency range from $5 \mathrm{kHz}$ to 100 $\mathrm{kHz}$. In considered materials, the specific losses by cycle, for an induction peak given $B_{\max }$, increase with the frequency and often in a considerable way [32]. Total losses per cycle behavior has a frequency relationship dependency, given by Bertotti as $P / f=B_{\max }^{3 / 2} \cdot f^{1 / 2}[33]$.

This attractive high-frequency behavior is due to low hysteresis loss and low eddy current loss concerned with the thin ribbon thickness and the high resistivity. The finemet alloy with high remanence ratio shows low core loss in high frequency range as compared with conventional high remanence ratio materials such as Febased amorphous and 50 wt. \% Ni Permalloy.

\section{Conclusion}

Three of the more important magnetic properties, permeability, coercive force, and hysteresis loss, are adversely affected by internal stress introduced during the process. Annealing can reduce this factor. The principal interest of material annealing under magnetic field is to change hysteresis loop. This procedure allows providing, for purposes of industrial applications, features special uses. The magnetization and magnetic losses dependencies of frequency, magnetic field were measured at room temperature in rapidly solidified ribbons and annealed ribbons. Magnetostriction experiments were made in the large annealing range. Nanocrystalline material annealed under longitudinal field has permeability $>10^{5}$. Of course, heat treatment under longitudinal field has designed to make the uniaxial anisotropy and improve hysteresis loop characteristics. We can estimate the size of grains for each annealing temperature $\mathrm{T}_{\mathrm{a}}$, using the model of Herzer, who gives a relationship $\mathrm{H}_{\mathrm{c}} \sim \mathrm{D}^{2 / 3}$. The great influence of annealing temperature on the reducing of coercivity leads to reducing the core loss. As expected, the influence of annealing on magnetostriction can be given by comparison to coercivity. The decrease of magnetostriction by annealing under longitudinal magnetic field allows obtaining preferentially uniaxial anisotropy.

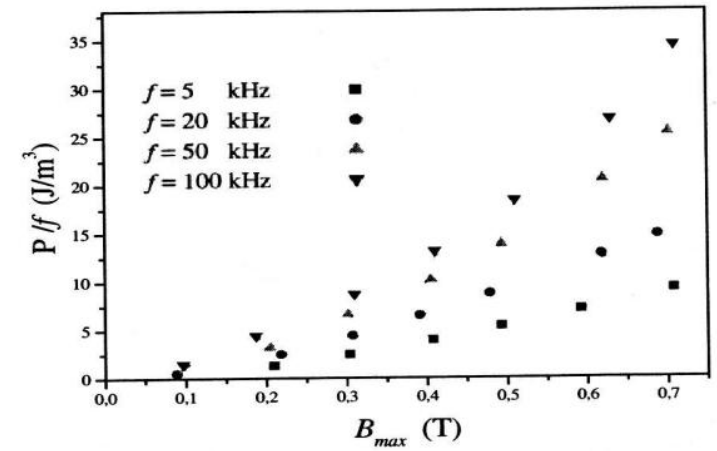

Fig. 5 Experimental losses by cycle of the nanocrystalline sample obtained at different Bmax for 5, 20, 50 and $100 \mathrm{kHz}$ frequencies.

\section{References}

1. C. Suryanarayana, Int. Mater. Rev. 40, 41 (1995)1.

2. B. Cantor, Mater. Sci. Forum 307, 143 (1999)

3. A.L. Greer, Mater. Sci. Forum 269, 3 (1998)

4. Y. Yoshizawa, S. Oguma, K. Yamauchi, J. Appl. Phys. 64, 6044 (1988)

5. K. Suzuki, N. Kataoka, A. Inoue, T. Masumoto, Mater. Trans. JIM 31, 743 (1990)

6. K. Suzuki, A. Makino, A. Inoue, T. Masumoto, J. Appl. Phys. 64, 33 (1993)

7. H. Lachowicz, A. Slawska-Waniewska, J. Magn. Magn. Mater. 133, 238 (1994)

8. A. Makino, T. Bitoh, A. Inoue, T. Masumoto, J. Appl. Phys. 81, 2736(1997)

9. M.A. Willard, D.E. Laughlin, M.E. MacHenry, D. Thoma, K. Sickafus, J.O. Cross, V.G. Harris, J. Appl. Phys. 84, 6773 (1998)

10. G. Herzer, IEEE Tras. Magn. MAG-25, 3327 (1989)

11. C. Beatrice, F. Vinai, G. Garra, P. Mazzetti, J. Phys.Colloques C8, 49, 1323 (1988)

12. Yoshizawa, Y. Mater. Sci. Forum 307, 51 (1999)

13. Herzer, G. IEEE Trans. Magn. 26, 1397 (1990)

14. Houssa R., V. Franco, A. Conde,J. Magn. Magn. Mater. 203, 199 (1999)

15. Gupta A.,N. Baghat, G. Principi, A. Maddalena, N. Malhotra, B.B. Dansannacharya, P.S. Goel, H. Amenitsch, S. Bernstorff, Intermetallics 8, 287 (2000)

16. P. Allia, M. Baricco, M. Knobel, P. Tiberto, F. Vinai, Mater. Sci. Eng. A 179\&180, 361 (1994)

17. P. Allia, P. Tiberto, M. Baricco, F. Vinai, Appl. Phys. Lett. 632759 (1993)

18. L.F. Barquin, J.C. Gomez Sal, P. Gorria, G.S. Garitaonandia, J.M. Barandiaran, J. Phys. : Condens. Matter. 10, 5027 (198)

19. M.S. Leu, T.S. Chin, I.C. Tung, C.M. Lee, Jpn. J. Appl. Phys. 38, 707 (1999)

20. T. Kulik, T. Horubala, H. Matyja, Mater. Sci. Eng. A157, 107 (1992)

21. Y. Yoshizawa, K. Yamauchi, IEEE Trans. Magn. 25, 3324 (1989)

22. Yoshizawa, Y, K. Yamauchi, J. Mag. Soc. Jpn. 14193 (1990)

23. M.L. Soltani, Journal of non-Crystalline Solids, 353, 2074 (2007)

24. M.L. Soltani, Journal of Physics: Conference Series, 98, $062014(2008)$

25. T. Kulik, J. Non-Crystal Solids 245, 145 (2001)

26. R.W. Cahn, Materials Science and Technology, 9,493 (VCH, Weinheim 1991)

27. T. Kulik, A. Hernando, Mater. Sci. Forum 179181, 587 (1995)

28. R. Alben, J.J. Becker, M.C. Chi, J. Appl. Phys. 49 1653 (1978)

29. G.Herzer, Proc.Int.Symp. on 3-D transition-semimetal thin films, Sendai, 130 (1991)

30. G.Herzer, J.Mater. Eng.Performance, 2, 193 (1993)

31. G.Herzer, . Magn. Magn. Mater. 157/158, 133(1996)

32. M.L. Soltani, A.M. Mansri, M. Lahoubi, Revue de Metallurgie (submitted)

33. G. Bertotti, IEEE Trans. On Mag. 24, nº1 (1988) 